\title{
Intercept Vertical Transmission-Perinatal Transmission of HIV
}

National Cancer Institute

\section{Source}

National Cancer Institute. Intercept Vertical Transmission-Perinatal Transmission of HIV.

NCl Thesaurus. Code C15808.

Develop, evaluate, and implement strategies for interrupting vertical transmission of HIV from mother to child and extend our understanding of perinatal transmission and the pathogenesis of early infection (treatment of children will be addressed in other objectives). (Note: The OAR Coordinating Committee on Therapeutics feels scientific objectives 3E through 3G are of equal weight in terms of scientific priorities.) 